\title{
Study to Explore the Effect and Thinking the Application of Chinese Painting in the Jade in the Professional Education
}

\author{
Guangzhao Yang \\ Hebei Academy of Fine Arts, Shijiazhuang Hebei, 050700, China
}

\begin{abstract}
Keywords: Nanyang, Caving jade professional teaching, Chinese painting, Function.
\end{abstract}
\begin{abstract}
With the further development of the the socialist market economy in new period. Government also stressed the importance of traditional culture industry, jade caving is have the further development opportunists in new period ; this article choose Nanyang area as an example, studied the current situation of jade craving education, analysis the impact of Chinese painting in the jade casting craving and hoping to promotion the improved of Jade carving and processional jade craving education by provide corresponding support and to promoting the improvement of local market and sustained and healthy development of jade.
\end{abstract}

\section{Introduction}

The jade and jade caving and Chinese painting are two main forms of traditional Chinese culture. Under the new the socialist market economy, importance of traditional culture and art work has been stress by government; the education of jade craving should leverage in the internal link between Chinese painting and jade craving; the healthy growth of jade craving art should be promoted by the interaction and blending of reference art, to increase enhance the teaching quality and crate market opportunities, and to make the risen of jade industry flourishing and achieve sustained and stable inheritance in modern society.

\section{Development of the professional teaching of jade caving in Nanyang}

The vigorous development of Nanyang jade industry gradually in the Nanyang region, in which Nanyang City is the cultural and industry center of the jade, which made great influence in the development, characteristic ,economy as well as cultural heritage influence to this area. In the new period, the jade craving major in Nanyang Normal University also in the development process of firmly in seize and opportunities to to optimize and adjust the teaching. In order to improve the quality of jade craving education, promote the local development of jade economic and cultural , make enormous contribution. But with the progress of the times, many jade craving majors are set by other universities, advantaged of Nanyang jade carving are gradually weakened, the reformation and development of education are showing down, the Nanyang jade industry also emerge in the some problems, such as lacking of vitality works, and low in artistic conception level. They had made certain bad influence in the development of Nanyang jade industrial and professional jade craving education and the cultural inheritance of jade culture [1]. Therefore, under the current social background, the reformation of Nanyang jade craving education and should focus on the topic of how to transfer the jade craving from industry craft to art piece while combine theory and practice combine together and introduce Chinese painting elements penetrated into jade in the creation, prompted the creation of jade in the beauty from the surface of the object into a real beauty of artistic conception, enhance the artistic value of jade in the works of art, and conduct professional training in jade craving and then promote the development of jade economics and culture contribute to the envelopment of traditional culture.

Compared with other general professional subjects, jade craving education has very strong practicality character, like art education, with similar focus of guiding hands-on operation ability, innovation ability and social practice ability of students, through the teaching of science and to 
significantly improved their jade carving professionalism. So in the new period, optimization and reformation of professional jade craving education should focus on the combination of theory and practice, as well as lectures work about production and processing of jade and train the students to high-quality jade craving talent,to meet the social expression demand. As the country's first professional jade craving education prevent, there is still many issues in the Nanyang jade craving major,the teaching system is still not perfect, unable to effectively cultivate students' innovation consciousness and creative ability,and not conducive to the students in terms of jade to obtain long-term development. So, it is very necessary to get some support from Chinese painting, deepen students understanding of Chinese traditional culture, perfect which Chinese painting in professional education fusion together, prompted the Chinese painting in the jade professional teaching role full play, for the inheritance of Chinese jade carving art contribution to the corresponding power.

\section{Issues in professional jade craving education}

As seen from the current basic situation jade craving, ether are many issue remain that can not conducive to the cultivation of teaching quality and enhance students' jade craving in the professional quality, but also produced some adverse effects to the development of jade industry in our country and the cultural heritage of jade culture. Some of the typical issues are shown below:

\section{The apprenticeship is the main teaching mode.}

Construction of jade caving teaching system in Nanyang area is still not perfect, professional formation and development is relatively slow, lacking affecting accouters in all aspects and attention,did not formed into a more scientific and reasonable arrangement and planning, no effective development of adverse impact on certain professional practice teaching reformation in the teaching practice. Apprenticeship is still the main teaching mode, students are in accordance with the master's teaching and philosophy of jade caving art creation. Their own personalized jade caving literacy can not effectively and can not cultivate students' professional knowledge, ability and the structure of the system is also often not perfect, restricted to enhance students' creative ability. Apprentice not conducive to optimizing the inheritance of [2] At the same time, in the jade in the professional teaching, teaching general to classroom practice based, students' creative ideas can not be effectively expand, can't even showed a clear theme in his works. Therefore, extremely easy to cause the creation of jade in the works lack of artistic charm, unable to appreciate bring certain aesthetic impact, limits the increase of the value of works of art.

\section{Shortage of teachers.}

The current high level of jade craving master mainly in the social operating instead of teaching in college. in the art circles, jade did not pay enough attention to school education, professional qualities do not deposits at all. Inheritance of jade craving master is not willing to teach in colleges and universities therefor resources for high quality teacher for colleges and universities choose from is very limited, eventually leading to lack of jade in the professional teaching team, severely restricted the development of modernization of jade in the professional, is not conducive to the jade cult re in the art in our society to inheritance.

\section{School environment construction is not perfect.}

Nanyang Normal University is the only one with undergraduate of jade crabbing in the education of the school, the teaching space is relatively small with limited supporting funds, renewal of equipment, lack of motivation, teaching resource is missing. At the same time, in the teaching environment construction, due to the lack of teaching resources; the school is not able to create good environment to the student, like classroom, practice; school enterprise joint work carried out by certain restriction, undergraduate teaching practice base construction is not ideal. On the students' practice ability culture and jade craving in the artistic accomplishment of the formation of adverse effects severely limits the jade in the industrialization of construction and development, is not conducive to the jade in the art in the current social context inheritance. 


\section{The role and penetration of Chinese painting in caving jade}

In order the promote further development of the jade craving educations, Chinese painting can be introduced inth the jade craving education as a new teach concept, Chinese painting's artistic creation thought can guide jade craving into the professional teaching, and to cultivate the students' art quality and aesthetic ability, encourage students to the creation of jade in the works of artistic value has been significantly improved, effectively promote the area jade craving in the industry for further development. Promote China jade craving in art in the current social context get good inheritance [3]. In the undergraduate based jade craving teaching practice, teachers not need to train students for jade craving in the creation by the theoretical, knowledge and practical skills, not also to cultivate the art thinking and innovation ability of students, guide students to integrate their own personal views in the creative process. And infiltration of Chinese painting education can in the aspects of thought and art to students to implement scientific education and guidance, promote students aesthetic ability has been significantly improved, also landscape painting, literati painting and painting etc. are different types of creation, but also to jade in the art provides rich subject matter, has the extremely important influence on jade craving $\mathrm{n}$ the professional teaching quality improvement. This article on the Chinese painting in the caving jade professional teaching in the specific analysis of the penetration

\section{The embodiment of "shape" and "god" in Chinese painting in caving jade Teaching.}

Shape "and" god "are important part of Chinese painting concept, but also constitute one of the core elements of the creation of it; in the current Chinese painting teaching, the general landscape, flower and bird and figure paintings, shape similarity and spirit of understanding and communication can be sublimated to the artistic value of painting works, and will" shape "and" god "of the idea is applied to works of jade craving in the creative process to make jade in the works from the development of modern arts and crafts to enrich the connotation of art works of art, jade in the industry create a broad space for development.

First of all, to strengthen the image of the art process modeling work attention. Chinese painting creation is bound to need to form, and the shape is the spirit of the work of the work of the spirit of the re transmission. The creation of Chinese painting into the jade in the professional teaching, students in the jade in the shape of the process should also combine Chinese painting creation thought, strengthening the understanding of the lyrics in the line, in the task in the process of carving strengthen the importance of the line change and attention are in carved flower to flower line performance growth posture, and then through the length, density and contrast, show the variability of the jade in the, flexible convey jade in the works to the ideological content of the performance.

Secondly, in the creative practice to explore the change of contrast.The teaching of Chinese painting will generally require students to choose between the traditional classic or modern society, more representative works to appreciate and analyze, and in contrast to found changes in the creation of Chinese painting, this change can be formed in the new period of the development process or creation rules changes; it can also be a solar term, climate change, or shades of wet and dry changes in the representation of the work and to convey the emotions and emotional changes. This teaching ideas into the jade in the teaching, teachers can require students to carefully observe the life and the life details of the changes and students to produce their own insights into works in, was a different kind of artistic effect, effectively enhance the student jade in the artistic creation of artistic value.

Integration of "Qi" and "Yun" in Chinese painting with caving jade professional teaching

Qiyun vivid in the artistic creation of Chinese painting is an important part of Sheikh six, but also the core idea of the Chinese Contemporary Chinese painting teaching, mainly reflected in Chinese painting should attach importance to the work connotation, the students created works of art should can reflect some thought. Qi is a kind of work spirit which is refined and developed from the artist's own accomplishment. In the specific creation, it can clearly show the physical and chemical forms of painting master and caving jade master. The rhyme is the charm of thought, works out the inner beauty. Is mainly a painter and jade in the master in the creation process, in clearly under the premise of its composition and meaning, good works to grasp the charm and the core spirit, make the work to 
show a unique charm and ensure the works of the ideological connotation can get maximum abundance.

Chinese painting "theory" and "law" in the caving jade professional teaching highlights.

No any orders without any rules, apply to the creating of Chinese painting creation art. Which means the creation need not only attention to the image, but also the spirit of, should also follow the creation method, only to follow the rationale of the heaven and the earth before they can ensure that works under the constraint of can get lasting inheritance. And in the caving jade professional teaching, to promote the caving jade art in modern society to get the corresponding inheritance and development, also should strengthen the attention to the theory of [5]. At the same time, it should pay attention to guide the students in the learning process of "teacher is not the teacher, that is to say, in the jade in the art teaching process on the theory and method of teaching should let the students in learning the ideas of teachers, observe things in life, and then combined with the actual life to cultivate and gradually formed their own personalized creative thinking, and ultimately the formation of unique writing style, encourage students to get better in the future development of. Only in this way can we truly cultivate high-quality caving jade professionals, for the caving jade art heritage brings new opportunities for development.

\section{Conclusion}

To sum up, Chinese painting creation and caving jade professional teaching has a certain commonality, Chinese painting will be integrated into the caving jade professional teaching can promote the caving jade professional teaching glow with exuberant vitality. Therefore, jade in the professional teaching must strengthen the importance of Chinese painting, appropriate combination of jade in the professional teaching into Chinese painting artistic thought, in training the students thinking of art and jade in the creative accomplishment based, jade in the industry of modernization and development provide new opportunity, make certain contribution for China's jade in the art of inheritance.

\section{References}

[1] Jiang Changsong. Jade ink Danqing -- on contemporary jade Tong Hui Juan caving works in, Chinese painting art carving phenomenon, 2015(7):86-86.

[2] Wang Ruiqiang. The role of Chinese painting in caving jade professional teaching and the thinking of, Art world ,2014(9):88.

[3] Luo Feifei. Relationship of Chinese painting and art jade caving, Journal of Xinjiang Vocational University, 2011,19(5):47-49.

[4] Luo Feifei. Dan--jade caving jade art inquiry, Jade International Symposium on Science .2011:353-357.

[5] Zhang Chunlei. On the Nanyang jade caving to China draw admiration and art reconstruction, Journal of Nanyang Normal University ,2015,14(11):42-45. 